\title{
Angiocentric glioma: a rare intractable epilepsy-related tumour in children
}

\author{
Wiesława Grajkowska ${ }^{1}$, Ewa Matyja², Paweł Daszkiewicz ${ }^{3}$, Marcin Roszkowski ${ }^{3}$, Jarosław Peregud-Pogorzelski ${ }^{4}$, \\ Elżbieta Jurkiewicz ${ }^{5}$ \\ ${ }^{1}$ Department of Pathology, The Children's Memorial Health Institute, Warsaw, ${ }^{2}$ Department of Clinical and Experimental \\ Neuropathology, Mossakowski Medical Research Centre Polish Academy of Sciences, Warsaw, ${ }^{3}$ Department of Neurosurgery, \\ The Children's Memorial Health Institute, Warsaw, ${ }^{4}$ Department of Pediatrics, Pediatric Hematology and Oncology, \\ Pomeranian Medical University, Szczecin, ${ }^{5}$ Department of Radiology, The Children's Memorial Health Institute, Warsaw, Poland
}

\begin{abstract}
Angiocentric glioma is a low-grade tumour that occurs in children and young adults with a long-standing epilepsy. The typical histopathological features of this tumour is the presence of spindle-shaped cells, radially oriented around the cortical blood vessels.

We present two teenage cases of the angiocentric variant of glioma: 1) a 15-year-old girl with a chronic and intractable partial epilepsy with cystic tumour located in the right temporal lobe and 2) a 14-year-old boy with intractable seizures and an extensive cortical lesion in the left parieto-occipital area. In both cases, the total tumours excision was performed. The histopathological findings revealed a characteristic angiocentric pattern that was composed of elongated cells arranging in pseudorosette-like structures around blood vessels. Moreover, schwannoma-like areas and subpial neoplastic infiltration with palisading of tumour cells at the brain surface were seen. The neoplastic cells displayed immunoreactivity for GFAP, S-100 protein and vimentin. A slight "dot-like" EMA staining, suggesting ependymal differentiation, was detected. The clinical and pathological picture allowed to establish the diagnosis of angiocentric gliomas. The patients were discharged home in a good condition and without seizures. During the 4-year follow-up, the tumour recurrence and seizures were not observed. The appropriate diagnosis of this peculiar type of usually low-grade glial tumour is important for adequate and successful treatment. The differential diagnosis requires the exclusion of other tumours with an angiocentric pattern, i.e. ependymoma, astroblastoma, which are associated with more aggressive biology.
\end{abstract}

Key words: angiocentric glioma, intractable epilepsy, paediatric brain tumour, schwannoma-like pattern.

\section{Introduction}

Angiocentric glioma (AG) is a rare, low-grade brain tumour in children and young adults with a long- standing epilepsy $[6,8]$. It has been included into the group of "other neuroepithelial tumours" in the current WHO Classification of Tumours of the Central Nervous System 2007 [3,5]. It is a slowly growing, 
indolent neoplasm, classified as WHO grade I. Angiocentric glioma occurs within a wide range of 2.3 70 years but it is mostly encountered in childhood and adolescence (mean age 17 years) [2].

Angiocentric gliomas usually develop in the cerebral hemispheres. Majority of them are located superficially in the frontal, temporal or parietal lobes, as well as in the hippocampal region. The case with features of angiocentric glioma in the posterior midbrain was also described [7]. Although AGs are typically localized within the cerebral cortex, they may extend into the adjacent white matter. On MR imaging, AGs are T2- and FLAIR hyperintense and hypointense on T1-weighted images, without contrast enhancement $[10,19]$. Calcifications are rarely encountered. More than $90 \%$ of patients with AG suffer from a long-standing epilepsy with intractable partial seizures. The diagnosis of AG is established by histopathological examination which should confirm the main angiocentric tumour pattern. Immunohistochemical study documented the glial origin of neoplastic cells with an expression of glial fibrillary acidic protein, S-100 protein and vimentin.

During the last years, the increasing histopathological and clinical data on angiocentric gliomas have been reported but the biology of this tumour is not enough understood $[2,4,9,18,19]$. We report two additional teenage cases of AGs with a well-documented long, 4-year follow-up.

\section{Cases report}

\section{Case 1}

A 15-year-old girl with a chronic and intractable partial epilepsy was admitted to the Neurosurgical Department of the Pomeranian Medical University in 2010. Psychomotor development was normal and familial history was insignificant. Physical examination did not reveal any focal deficits or signs of increased intracranial pressure. EEG showed the presence of fairy regular basic activity with series of slow waves (frequency 3.3-4 Hz and voltage $120 \mathrm{mkV}$ ) in the frontal-occipital-temporal lobes of the right hemisphere with tendency for generalization. Ophthalmological examination revealed a normal disc of the optic nerve with distinct borders within the fundus. MRI scan revealed a well-delineated, cystic lesion, measuring $12 \times 20 \times 20 \mathrm{~mm}$, located within the middle areas of the temporal lobe of the right hemisphere (Fig. 1A). The lesion did not show any enhancement after contrast administration. Surgical treatment was performed with a total tumour excision. The histopathological diagnosis was angiocentric glioma. Control MRI head scan performed 8 months following the surgery did not detect any relapse. Axial FLAIR and postcontrast T1-weighted images showed the resection cavity with a small hyperintensive area, probably corresponding with gliosis, without contrast enhancement (Figs. 1B-C). The patient was discharged home
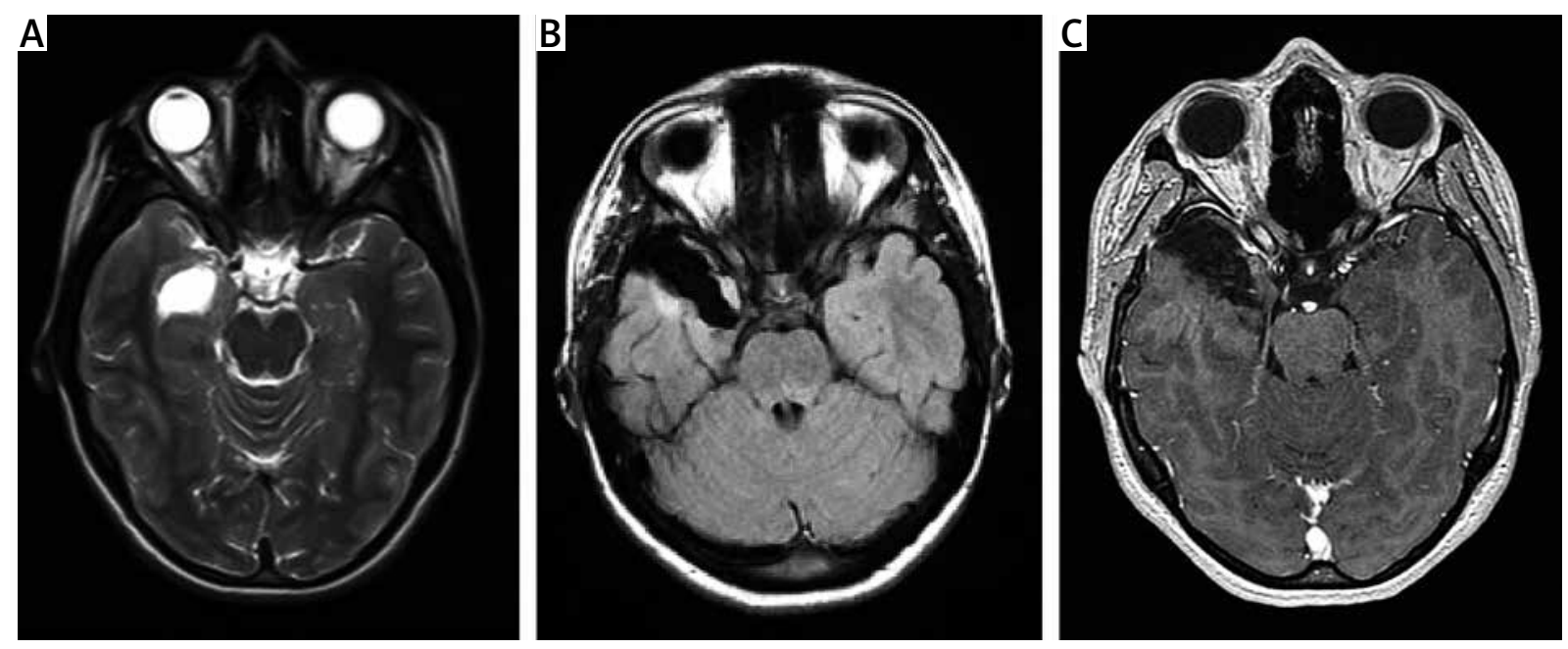

Fig. 1. MRI feature. Case 1. A) Preoperative MR brain examination. Axial T2-weighted image showing a well-delineated hyperintense lesion located in the right temporal lobe. B, C) Post-operative MR images. Axial FLAIR (B) and postcontrast T1-weighted (C) images showing a resection cavity with the small hyperintensive area - gliosis. No contrast enhancement. 
in a good condition with no seizure recurrence. Due to abnormal EEG the patient was on long-term carbamazepine $2 \times 200 \mathrm{mg} / \mathrm{d}$ treatment.

\section{Case 2}

A 14-year-old boy was admitted to the Department of Neurosurgery of the Children's Memorial Health Institute. He had a history of prematurity and developed his first generalized seizure at the age of 3. Thereafter, his psychosomatic development progressed normally, except for a mild horizontal nystagmus. Since the age of 11 , he presented the simple focal seizures (paroxysmal right-sided hemianopsia, monoparesis of the left leg with auras in the form of scintillating scotomas) at the mean rate of 5 per day. The first imaging studies (CT) revealed an extensive lesion within the medial structures of left parieto-occipital lobes. The ventricular system was symmetrical and not enlarged. MRI revealed the irregular area of signal intensity on T2-weighted and FLAIR images (Fig. 2A) and T1-weighted with only a slight enhancement of a part of the tumour (Fig. 2B). HMRS spectrum revealed a slight elevation of the choline peak and slight depression of the $\mathrm{N}$-acetyl-aspartate (NAA) peak. Overall, MR appearance was consistent with focal cortical dysplasia or benign tumour. EEG study revealed moderate focal discharges in the left temporo-occipital and parietal areas, with a slight tendency to generalize over the entire ipsilateral and posterior aspects of contralateral hemispheres. Discharges were enhanced by hyperventilation and somnolence. Moderate clinical response was obtained upon application of anti-epileptic medication (valproic acid and levetiracetam). At admission to the Department of Neurosurgery, he was in a good overall condition with no neurological deficits. The left-sided parieto-occipital craniotomy was performed under neuronavigation guidance and the lesion within the parietal lobe, parieto-occipital fissure and crus of the hippocampus was totally excised. The histopathological diagnosis was angiocentric glioma. After surgery, the patient presented right-sided hemianopsia with no other neurological deficits. Post-operative MRI study revealed a well-delineated resection cavity with hyperintensity at its posterior margin, probably corresponding with gliosis, on axial FLAIR (Fig. 2C) and post contrast T1-weighted images. There was no contrast enhancement. Pre-discharge CT revealed a postoperative site with a moderate-sized pericerebral hematoma with no mass effect and no disturbances of cerebral circulation. He was discharged home in a good overall condition, fully mobile and fit. The patient has been without tumour recurrence and seizures for 4-year follow-up.

\section{Material and methods}

The surgical material was prepared by standard methodology. Biopsy tissue was fixed in $10 \%$ neu-
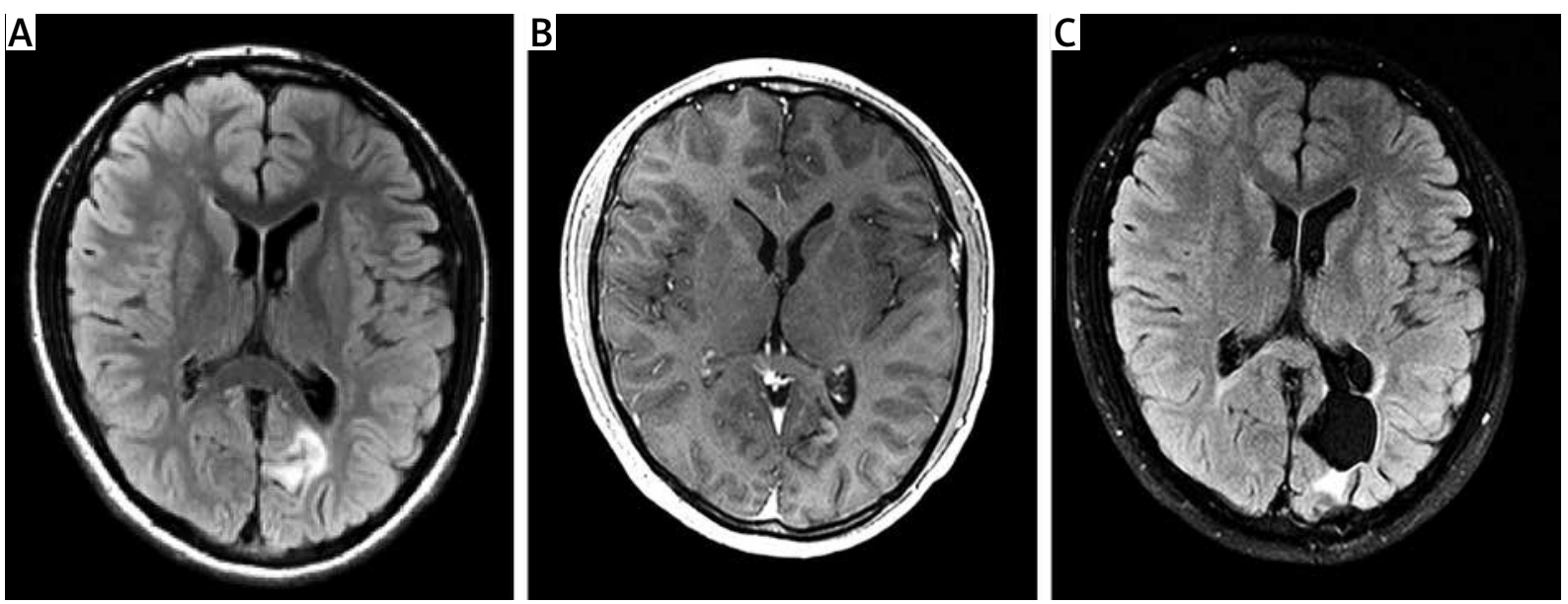

Fig. 2. MRI feature. Case 2. A) Preoperative MR brain examination. Axial FLAIR image showing a corticosubcortical mass with high signal intensity, involving the left parieto-occipital lobes, without mass effect. B) Preoperative MR brain examination. Contrast-enhanced axial T1-weighted image at the same level showing a slight enhancement of a part of the tumour. C) Post-operative MR images. Axial FLAIR image showing a well-delineated resection cavity. 
tral-buffered formalin, embedded in paraffin blocks, sectioned at $5 \mu \mathrm{m}$ thick slices, and stained with hematoxylin-eosin (H\&E). Immunohistochemistry was performed on paraffin-embedded specimens; labelling was carried out by the avidin-biotin complex $(A B C)$ method with 3,3'-diaminobenzidine (DAB) as a chromogen. Primary antibodies were used against glial fibrillary acidic protein (GFAP; dilution $1: 800$ ), S-100 protein (dilution $1: 500$ ), epithelial membrane antigen (EMA; dilution $1: 100$ ), vimentin (dilution $1: 100$ ), synaptophysin (dilution $1: 200$ ), CD56 (dilution $1: 100$ ), cytokeratin-AE1/AE3 (dilution $1: 400$ ), and Ki67 (clone MIB-1, dilution $1: 100$ ). All antigens were obtained from Dako.

\section{Histopathological findings}

In both cases, histopathological studies of biopsy material revealed glial tumours composed predominately of elongated cells, aggregating around blood vessels and forming pseudorosette-like structures that closely resembled those of ependymoma (Figs. 3A-B). The neoplastic cells were mostly spindle-shaped, bipolar with eosinophilic cytoplasm and oval to elongate nuclei with irregular nuclear contours and granular chromatin. Moreover, the palisading pattern of tumour cells, mimicking schwannoma-like areas, was focally found (Figs. 3C-D). Compact, elongated cells were also seen in the superficial layers of cerebral cortex (Fig. 4E). Infiltration with palisade arrangement of the neoplastic cells was observed in the subpial areas at the brain surface (Fig. 3F). Mitotic figures were absent and necroses or vascular proliferation were not seen.

\section{Immunohistochemical staining}

The neoplastic cells displayed variable immunoreactivity for GFAP (Fig. 4A) and vimentin (Fig. 4B). The tumour cells were also immunopositive for S-100 protein (Fig. 4C). Moreover, the "dot-like" intracytoplasmic EMA positivity could be identified (Fig. 4D). The neoplastic cells were essentially negative for neuronal markers (synaptophysin, CD56) and cytokeratin AE1/AE3. The overall MIB-1 labelling index was low, less than $1 \%$.

The prominent angiocentric pattern of tumours growth allowed to establish the diagnosis of angiocentric gliomas.

\section{Discussion}

Angiocentric glioma is a rare, clinico-pathological entity that presents particularly in a childhood, often in association with medically intractable epilepsy [5, $6,8,18]$. The appropriate diagnosis of this entity is critical, as this tumour is usually slow-growing and treatable by surgical excision alone [1,18]. AGs are sometimes associated with adjacent foci of cortical dysplasia, thus careful pre-surgical evaluation of the lesion is necessary to plan the extent of resection and achieve a better seizure-free outcome $[11,20]$. Such association with coexistent malformation of cortical development/cortical dysplasia might suggest a developmental basis of their origin [14].

AG displays various histopathological features, which resemble ependymoma, astroblastoma, pilocytic/pilomyxoid astrocytoma and schwannoma. It is particularly difficult to distinguish AG from other gliomas that exhibit perivascular arrangement of neoplastic cells. Angiocentric glioma is focally composed of spindle cells with eosinophilic cytoplasm arranged around cortical and white matter blood vessels, mimicking ependymal or astroblastomatous pseudorosettes. The case with atypical histopathology including astroblastoma-like features and onionlike structures were described [16]. All sizes of vessels are involved, including large arteries of cortical surface as well as more deeply situated capillaries. The proper classification of angiocentric glioma and separation it from other similar entities might be particularly difficult in limited biopsy material.

The cases presented here displayed characteristic perivascular orientation of neoplastic cells, accompanied by a schwannoma-like component and subpial spreading. The glial origin of the tumours was confirmed by cytoplasmic GFAP immunoreactivity. In both cases, the neoplastic cells revealed EMA staining of a dot-like pattern. The presence of pseudorosette-like fashion around blood vessels and intracytoplasmic "dot-like" EMA immunostaining are characteristic of ependymoma and might be related with ependymal differentiation of neoplastic cells. It has been documented that the specific "dot-like" EMA staining corresponds to microlumens. The presence of microlumens and microvilli, typical of ependymal cells, has been described in some electron microscopic evaluations of AG cases $[15,18]$. These findings supported ependymal differentiation in angiocentric gliomas, thus the distinction of AG from classic ependymoma ought to be very carefully validated. Ependymoma is usually more monomorphic and sometimes exhibits true central-lumen rosettes, ependymal canals and dis- 

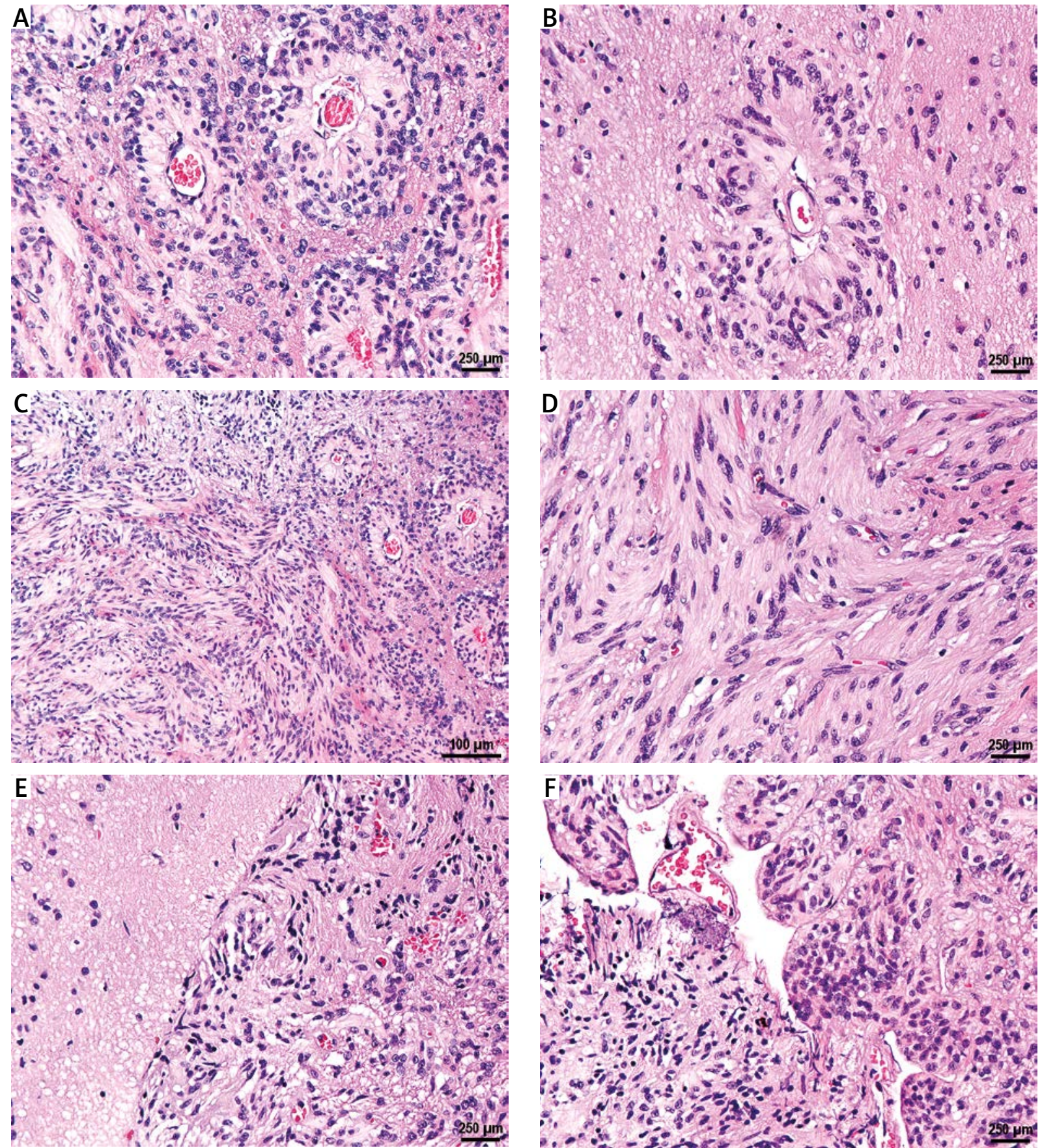

Fig. 3. Histopathological features of tumours. A, B) Angiocentric growth pattern with perivascular pseudorosettes, mimicking ependymoma. C) Compact masses of elongated neoplastic cells, mimicking schwannoma-like pattern. D) Palisade arrangement of the spindle-shaped tumour. E) Compact, elongated cells infiltrating the superficial layers of cerebral cortex. F) Subpial orthogonal aggregation of tumour cells in the subpial region of the cortex. H\&E.

tinct fibrillary cytoplasmic processes. Its extraventricular, occasionally superficial location is unique and a long history of seizures is not typical. Moreo- ver, the ependymoma is usually well-circumscribed, whereas angiocentric glioma presented more infiltrative growth. The correct identification of these 

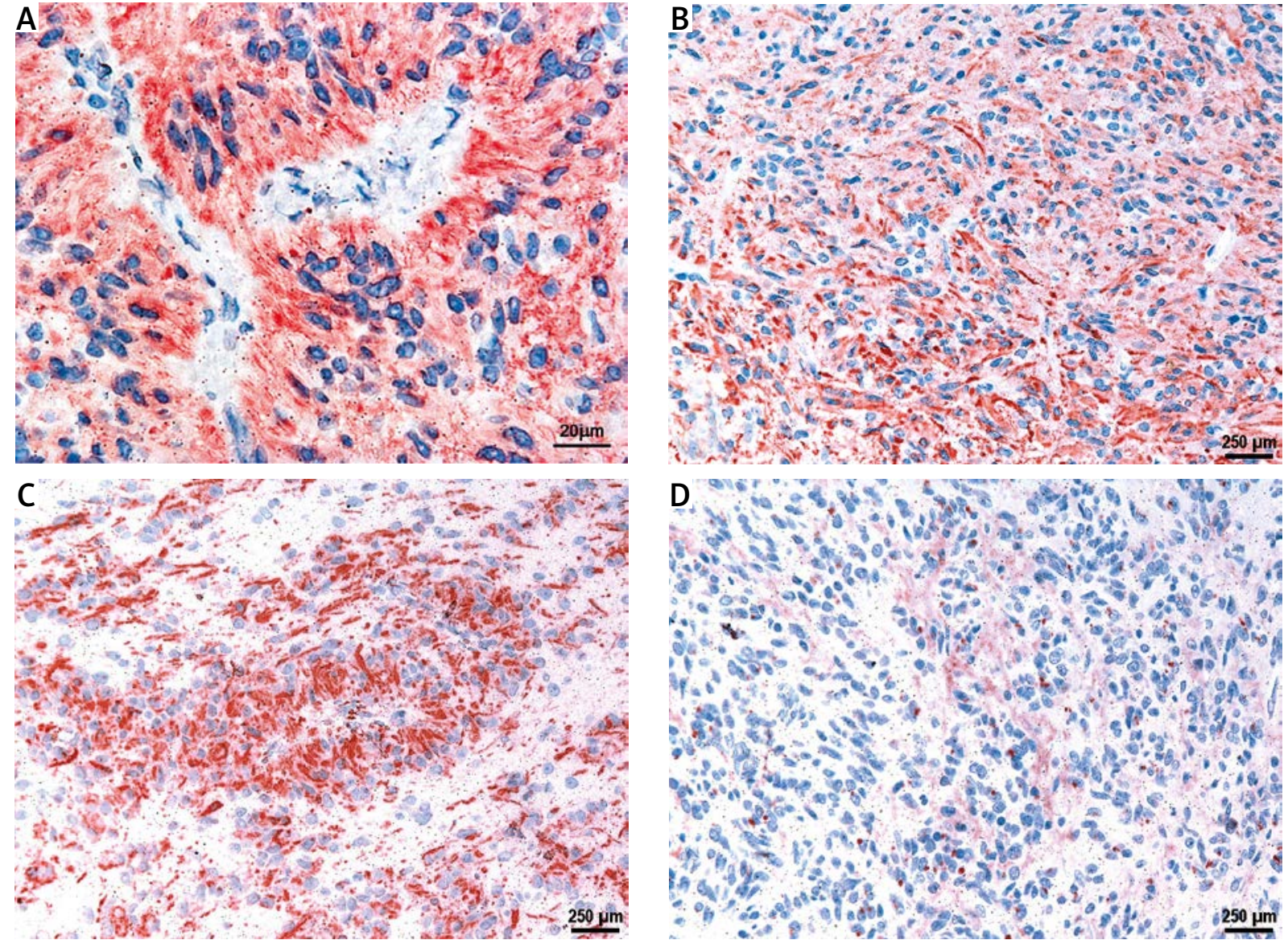

Fig. 4. Immunohistochemical staining. A) Strong cytoplasmic immunoreactivity for GFAP in bipolar tumour cells. B) Immunohistochemical stain for vimentin. C) S-100 protein immunoreactivity. D) Intracytoplasmic "dot-like" staining for EMA.

two brain tumours is crucial for their prognosis and treatment. However, the evidence of admixed features of these both entities might suggest their close relationship [13,21].

Angiocentric glioma may also resemble pilocytic astrocytoma, especially its pilomyxoid variant. However, pilocytic tumours are usually well-defined with myxoid background and their perivascular formations are not so distinct and compact as it is observed in angiocentric gliomas.

Other tumours of different histogenesis as astroblastoma and schwannoma ought to be also included in differential diagnosis. The distinction between AG and astroblastoma was a challenge as both tumours are characterized by a angiocentric growth pattern and strong GFAP expression. However, astroblastoma is a rare and controversial glia-derived neoplasm that is not universally accepted as a distinct entity. The so-called astroblastoma typically exhibits marked hyalinization of the blood vessels which is not characteristic of AG.

Another misleading feature of $A G$ is palisading arrangement of neoplastic cells at the brain surface that resemble schwannoma-like structures. Less frequently, the neoplastic cells may be arranged in solid nests or nodules. Mitotic figures are usually not apparent but in some cases the increased mitotic activity accompanied by malignant features i.e. microvascular proliferation and necroses has been described $[12,17]$. In such high grade variant of AG with malignant features and tendency to recurrence, the postoperative radio- or chemo-therapy ought to be considered.

The histogenesis of AG is controversial. Angiocentric growth, "dot-like" EMA positivity and ultrastructural features suggest the ependymal differentiation of neoplastic cells. An origin from radial glial cells has been proposed. The molecular features connect- 
ing with IDH1, IDH2 and BRAFV600E mutational status were described in three paediatric cases of this rare entity [4]. The loss of chromosomal bands $6 q 24$ to q25 was evidenced in 1 of 8 analyzed cases [18].

Angiocentric glioma is an epileptogenic tumour and might be associated with adjacent cortical dysplasia. The outcome of majority of AG patients is very good with freedom from seizures following a gross total resection. Our two cases with a long follow-up illustrated that a complete excision leads to surgical cure and provides a seizure-free outcome.

\section{Disclosure}

\section{Authors report no conflict of interest.}

\section{References}

1. Alexandru D, Haghighi B, Muhonen MG. The treatment of angiocentric glioma: case report and literature review. Perm J 2013; 17: e100-102.

2. Arsene D, Ardeleanu C, Ogrezeanu I, Danaila L. Angiocentric glioma: presentation of two cases with dissimilar histology. Clin Neuropathol 2008; 27: 391-395.

3. Brat DJ, Scheithauer BW, Fuller GN, Tihan T. Newly codified glial neoplasms of the 2007 WHO Classification of Tumours of the Central Nervous System: angiocentric glioma, pilomyxoid astrocytoma and pituicytoma. Brain Pathol 2007; 17: 319-324.

4. Buccoliero AM, Castiglione F, Degl'innocenti DR, Moncini D, Spacca B, Giordano F, Genitori L, Taddei GL. Angiocentric glioma: clinical, morphological, immunohistochemical and molecular features in three pediatric cases. Clin Neuropathol 2013; 32: 107-113.

5. Burger PC, Jouvet A, Preusser M, Hans VH, Rosenblum MK, Lellouch-Tubiana A. Angiocentric glioma. In: Louis DN, Oghaki H, Wiesttler D, Cavenee WK (eds.). WHO Classification of Tumors of the Central Nervous System. $4^{\text {th }}$ ed. IARC Press, Lyon 2007, pp. 92-93.

6. Chen G, Wang L, Wu J, Jin Y, Wang X, Jin Y. Intractable epilepsy due to angiocentric glioma: A case report and minireview. Exp Ther Med 2014; 7: 61-65.

7. Covington DB, Rosenblum MK, Brathwaite CD, Sandberg DI. Angiocentric glioma-like tumor of the midbrain. Pediatr Neurosurg 2009; 45: 429-433.

8. Fulton SP, Clarke DF, Wheless JW, Ellison DW, Ogg R, Boop FA. Angiocentric glioma-induced seizures in a 2-year-old child. J Child Neurol 2009; 24: 852-856.

9. Koral K. Angiocentric glioma. J Neurosurg Pediatr 2013; 12: 666.

10. Koral K, Koral KM, Sklar F. Angiocentric glioma in a 4-year-old boy: imaging characteristics and review of the literature. Clin Imaging 2012; 36: 61-64.

11. Liu CQ, Zhou J, Qi X, Luan GM. Refractory temporal lobe epilepsy caused by angiocentric glioma complicated with focal cortical dysplasia: a surgical case series. I Neurooncol 2012; 110: $375-$ 380.
12. Lu JQ, Patel S, Wilson BA, Pugh J, Mehta V. Malignant glioma with angiocentric features. J Neurosurg Pediatr 2013; 11: 350-355.

13. Lum DJ, Halliday W, Watson M, Smith A, Law A. Cortical ependymoma or monomorphous angiocentric glioma? Neuropathology 2008; 28: 81-86.

14. Marburger T, Prayson R. Angiocentric glioma: a clinicopathologic review of 5 tumors with identification of associated cortical dysplasia. Arch Pathol Lab Med 2011; 135: 1037-1041.

15. Miyata H, Ryufuku M, Kubota Y, Ochiai T, Niimura K, Hori T. Adult-onset angiocentric glioma of epithelioid cell-predominant type of the mesial temporal lobe suggestive of a rare but distinct clinicopathological subset within a spectrum of angiocentric cortical ependymal tumors. Neuropathology 2012; 32: 479-491.

16. Ni HC, Chen SY, Chen L, Lu DH, Fu YJ, Piao YS. Angiocentric glioma: A report of nine new cases, including four with atypical histological features. Neuropathol Appl Neurobiol 2014; doi: 10.1111/nan.12158 [Epub ahead of print].

17. Pokharel S, Parker JR, Parker JC, Jr., Coventry S, Stevenson CB, Moeller KK. Angiocentric glioma with high proliferative index: case report and review of the literature. Ann Clin Lab Sci 2011; 41: 257-261.

18. Preusser M, Hoischen A, Novak K, Czech T, Prayer D, Hainfellner JA, Baumgartner C, Woermann FG, Tuxhorn IE, Pannek HW, Bergmann M, Radlwimmer B, Villagran R, Weber RG, Hans VH. Angiocentric glioma: report of clinico-pathologic and genetic findings in 8 cases. Am J Surg Pathol 2007; 31: 1709-1718.

19. Shakur SF, McGirt MJ, Johnson MW, Burger PC, Ahn E, Carson BS, Jallo GI. Angiocentric glioma: a case series. J Neurosurg Pediatr 2009; 3: 197-202.

20. Takada S, Iwasaki M, Suzuki H, Nakasato N, Kumabe T, Tominaga T. Angiocentric glioma and surrounding cortical dysplasia manifesting as intractable frontal lobe epilepsy - case report. Neurol Med Chir (Tokyo) 2011; 51: 522-526.

21. Wang M, Tihan T, Rojiani AM, Bodhireddy SR, Prayson RA, lacuone JJ, Alles AJ, Donahue DJ, Hessler RB, Kim JH, Haas M, Rosenblum MK, Burger PC. Monomorphous angiocentric glioma: a distinctive epileptogenic neoplasm with features of infiltrating astrocytoma and ependymoma. J Neuropathol Exp Neurol 2005; 64: 875-881. 